\title{
Metal Processing with Ultra-Short Laser Pulses
}

P. S. Banks, M. D. Feit, A. M. Komashko, M. D. Perry, A. M. Rubenchik, and B. C. Stuart

This article was submitted to

High Power Laser Ablation, Santa Fe, New Mexico, April 23-28, 2000

\section{May 1, 2000}

U.S. Department of Energy

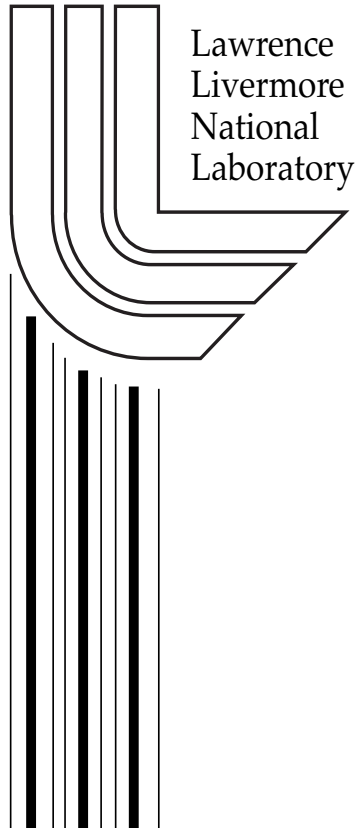




\section{DISCLAIMER}

This document was prepared as an account of work sponsored by an agency of the United States Government. Neither the United States Government nor the University of California nor any of their employees, makes any warranty, express or implied, or assumes any legal liability or responsibility for the accuracy, completeness, or usefulness of any information, apparatus, product, or process disclosed, or represents that its use would not infringe privately owned rights. Reference herein to any specific commercial product, process, or service by trade name, trademark, manufacturer, or otherwise, does not necessarily constitute or imply its endorsement, recommendation, or favoring by the United States Government or the University of California. The views and opinions of authors expressed herein do not necessarily state or reflect those of the United States Government or the University of California, and shall not be used for advertising or product endorsement purposes.

This is a preprint of a paper intended for publication in a journal or proceedings. Since changes may be made before publication, this preprint is made available with the understanding that it will not be cited or reproduced without the permission of the author.

This report has been reproduced directly from the best available copy.

Available electronically at http://www.doc.gov/bridge

Available for a processing fee to U.S. Department of Energy

And its contractors in paper from

U.S. Department of Energy

Office of Scientific and Technical Information

P.O. Box 62

Oak Ridge, TN 37831-0062

Telephone: (865) 576-8401

Facsimile: (865) 576-5728

E-mail: reports@adonis.osti.gov

Available for the sale to the public from

U.S. Department of Commerce

National Technical Information Service

5285 Port Royal Road

Springfield, VA 22161

Telephone: (800) 553-6847

Facsimile: (703) 605-6900

E-mail: orders@ntis.fedworld.gov

Online ordering: http:/ / www.ntis.gov/ordering.htm

\section{OR}

Lawrence Livermore National Laboratory

Technical Information Department's Digital Library

http: / / www.llnl.gov/tid/Library.html 


\title{
Metal processing with ultra-short laser pulses.
}

\author{
P. S. Banks, M. D. Feit, A. M. Komashko, M. D. Perry, A. M. Rubenchik, and B. C. Stuart. \\ Laser Program, Lawrence Livermore National Laboratory, P.O. Box 808, L-477, Livermore, CA 94550 \\ USA (925) 422-6131, fax (925) 422-5537, rubenchik1@1lnl.gov
}

\begin{abstract}
Femtosecond laser ablation has been shown to produce well-defined cuts and holes in metals with minimal heat effect to the remaining material. Ultrashort laser pulse processing shows promise as an important technique for materials processing. We will discuss the physical effects associated with processing based experimental and modeling results.

Intense ultra-short laser pulse (USLP) generates high pressures and temperatures in a subsurface layer during the pulse, which can strongly modify the absorption. We carried out simulations of USLP absorption versus material and pulse parameters. The ablation rate as function of the laser parameters has been estimated.

Since every laser pulse removes only a small amount of material, a practical laser processing system must have high repetition rate. We will demonstrate that planar ablation is unstable and the initially smooth crater bottom develops a corrugated pattern after many tens of shots. The corrugation growth rate, angle of incidence and the polarization of laser electric field dependence will be discussed. In the nonlinear stage, the formation of coherent structures with scales much larger than the laser wavelength was observed. Also, there appears to be a threshold fluence above which a narrow, nearly perfectly circular channel forms after a few hundred shots. Subsequent shots deepen this channel without significantly increasing its diameter.
\end{abstract}

The role of light absorption in the hole walls will be discussed.

Keywords: Femtosecond lasers, laser machining, materials processing, laser-matter interaction

\section{INTRODUCTION}

The use of femtosecond lasers allows materials processing of practically any material with extremely high precision and minimal collateral damage. Advantages over conventional laser machining (using pulses longer than a few tens of picoseconds) are realized by depositing the laser energy into the electrons of the material on a time scale short compared to the transfer time of this energy to the bulk of the material, resulting in increased ablation efficiency and negligible shock or thermal stress. The improvement in the morphology by using femtosecond pulses rather than nanosecond pulses has been studied in numerous materials from biological materials to dielectrics to metals. ${ }^{1-7}$

As part of our short pulse material processing studies, we modeled physical phenomena related to material processing. Our descriptions include one pulse ablation, cumulative effects of multiple pulses on the crater bottom, and light interactions with the channel walls. The penetration of laser radiation into the metal is very small and the ablation can be treated locally as onedimensional. We used the hydrocode $\mathrm{HYADES}^{8}$ to model ablation. We were able to evaluate absorption as a function of laser parameters and to suggest a reasonable estimate of the ablation rate. ${ }^{9}$ We start with an overview of these results. Then we will show how to match this initial ablation stage to a description of the plume over longer time scales.

Experiments show that the crater bottom becomes corrugated very quickly. We will explain the appearance of the corrugation and estimate the time needed for growth of relief. The absorption in the walls is essential for high aspect ratio drilling. We will discuss this problem in the last part of the paper. In the conclusion, we will summarize our understanding of the process of metal drilling and will formulate key problems still needing to be resolved. 


\section{PLANE ABLATION DESCRIPTION}

The laser radiation incident on the metal surface is absorbed in a very thin skin depth (less than $10 \mathrm{~nm}$ for $826 \mathrm{~nm}$ radiation in Al). Even for modest radiation fluxes of few $\mathrm{J} / \mathrm{cm}^{2}$, the concentration of absorbed energy in such a thin layer generates pressures in the range of a few megabars. At this high energy density, energy is transported out of the absorption zone by shock waves; typical expansion velocities are so high (few $\mathrm{km} / \mathrm{sec}$ ) that the density in the skin layer changes significantly in a time of a few tens of femtoseconds. Hence, adequate modeling of USLP laser-metal interactions requires use of a hydrocode with an electromagnetic wave solver to enable treating the modification of absorption during the pulse. The present work used the code HYADES which is described in detail in Ref. 8. Fig.1 presents modeling results for USLP absorption by an Al target in comparison with the experimental data of Milchberg et al. ${ }^{10}$ The pulse duration was $400 \mathrm{fs}$, and the laser wavelength was $308 \mathrm{~nm}$. The simulation reproduces the experimental data reasonably well. Differences exist, in part, because our calculations didn't take into account variations of refractive index with density and temperature nor increases of absorption due to melting of Al. Note the difference between simulations and experiment at low intensities. This implies that the "cold" values of refractive index used in the simulations did not precisely correspond to the specific material used in the experiment. In specific cases, models can be adjusted to agree better with experiment, but the present form is adequate to study the basic physics of USLP metal ablation.

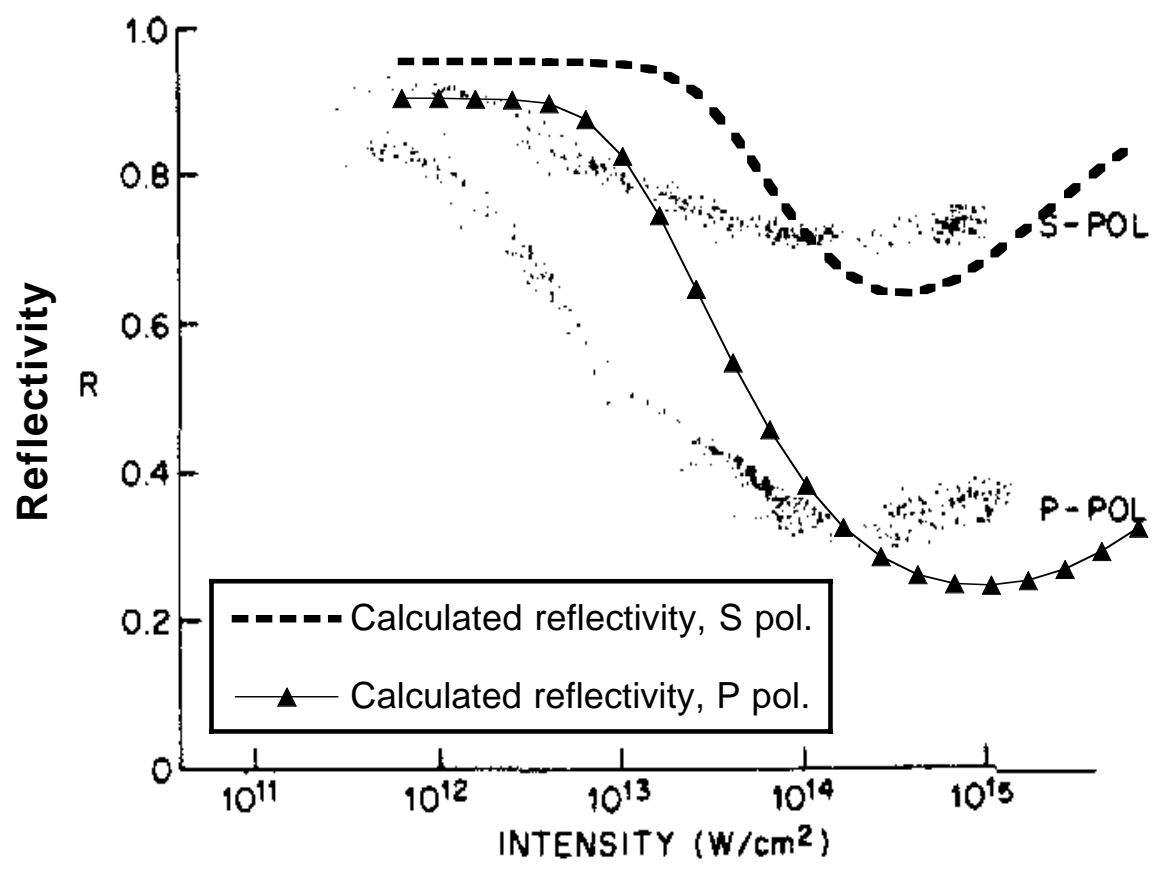

Fig. 1 : Comparison of theoretical and experimental absorption. Experimental data is from Milchberg et. al. ${ }^{10}$

Fig.1 indicates that absorption first increases with intensity and then drops at very high intensities. This behavior is generic for highly reflective metals. The absorption increase is related to plasma formation and expansion; absorption increases when the plasma density drops and gradients become less steep. At high intensities, the plasma becomes hotter, the collisional rate drops, and the reflectivity consequently increases. This "plasma mirror" effect has been observed and utilized in experiments with the Petawatt laser.

The calculated angular dependence of absorption in Al for different intensities and polarization are presented in Fig.2. One sees that at high intensities the plasma-enhanced absorption has an angular dependence similar in form to that of the cold metal-absorption is more efficient for P-polarized light. 


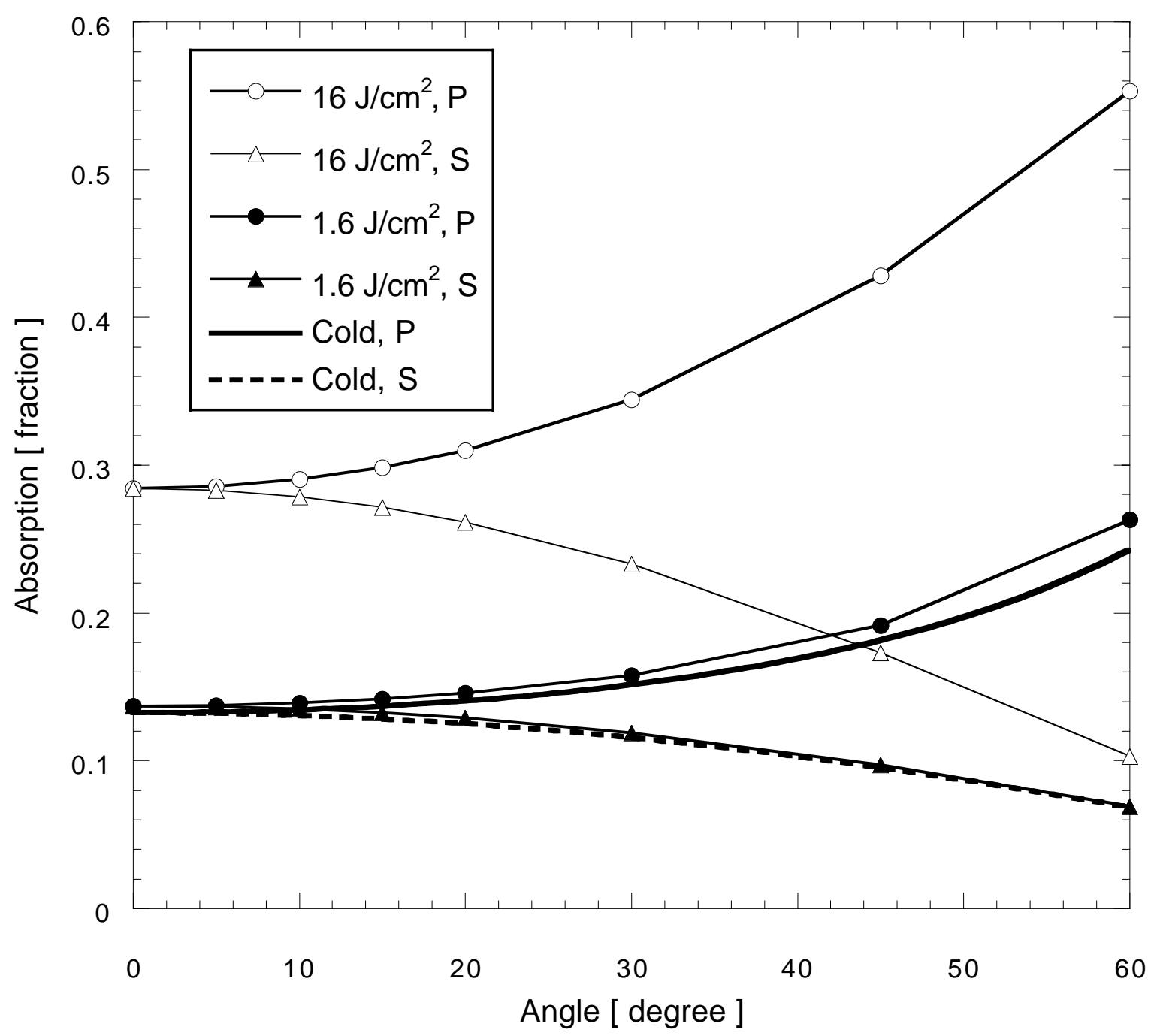

Fig. 2 : Calculated USLP absorption in Al as a function of angle of incidence and polarization $(\lambda=826 \mathrm{~nm}, \tau=150 \mathrm{fs})$.

One of the most important quantities to model is the amount of material removed by USLP ablation. Material removal takes place on a time scale much longer than that of energy deposition. The simulation of such long time behavior must include 3D geometrical effects, description of phase transition with significant changes of material density, descriptions of material strength etc. Such comprehensive theoretical descriptions are extremely difficult to realize. We recently suggested ${ }^{9}$, a plausible theoretical measure for the amount of material removed. If our calculations are continued to times of a nanosecond or longer, one notices that the amount of material moving away from the bulk is no longer changing. Therefore, the position of zero velocity is taken to divide material that will ultimately be ablated from that which will remain. Such an approach disregards, for a example, long time evaporation effects. But for metals, which cool rapidly, such effects are not important. Fig. 3 presents results of typical calculations. One sees that the steady position of the zero velocity point onsets at about $0.5 \mathrm{~ns}$ and afterward scarcely changes. Fig. 4 shows the removal rate as a function of absorbed energy calculated in this way. 


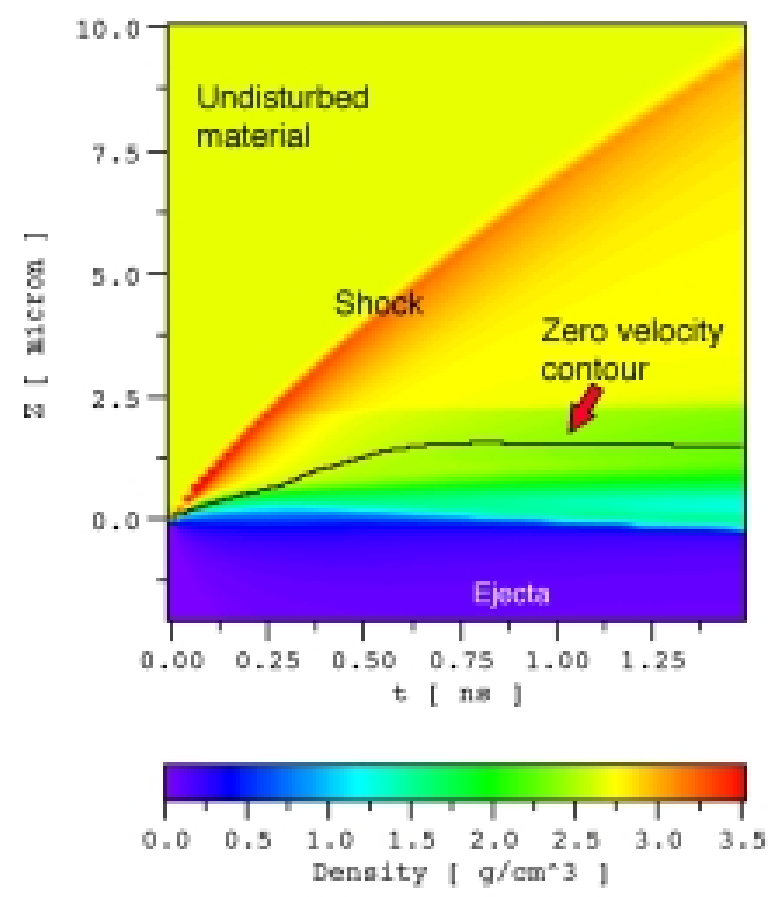

Fig. 3 : Zero velocity criteria description.

Our calculations indicate that for a wide range of absorbed energy Q, the removal rate varies as a power function of Q. The removal rate is presented as function of pulse duration in Fig.5a. One can see the sharp drop in removal efficiency, the amount of material removed by one pulse, for pulses with duration longer than one picosecond. Simultaneously, a sharp increase of total energy absorption takes place. For longer pulses of equal energy, the plasma expands enough to shield the ablation surface while the plasma profile is smooth enough to provide strong collisional absorption. Nearly all of the absorbed energy is carried out with the ejecta and does not effect the ablation surface.

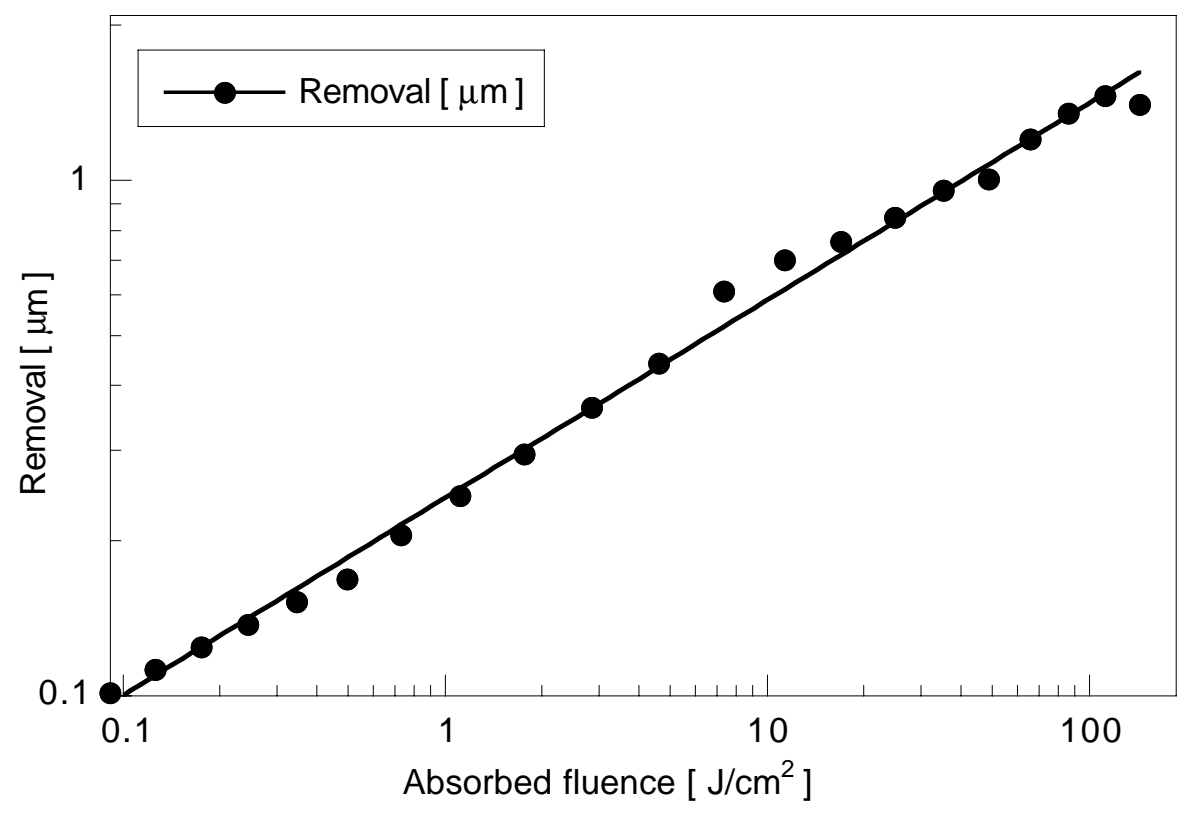

Fig. 4: Calculated removal rate versus absorbed energy ( Aluminum, normal incidence, $\lambda=826 \mathrm{~nm}, \tau=150 \mathrm{fs}$ ) . 


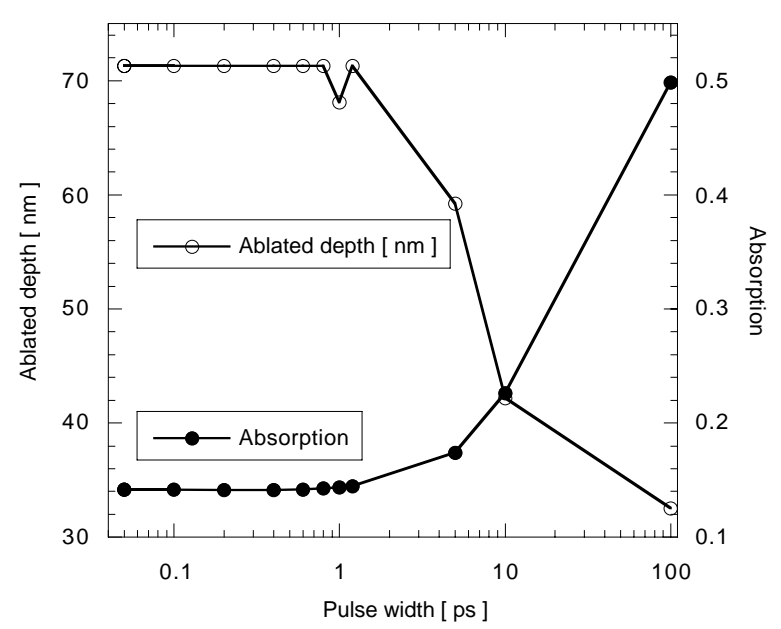

Fig 5a : Calculations of absorption and removal as a function of pulse length ( Aluminum, normal incidence, $\left.2 \mathrm{~J} / \mathrm{cm}^{2}, \lambda=826 \mathrm{~nm}\right)$.

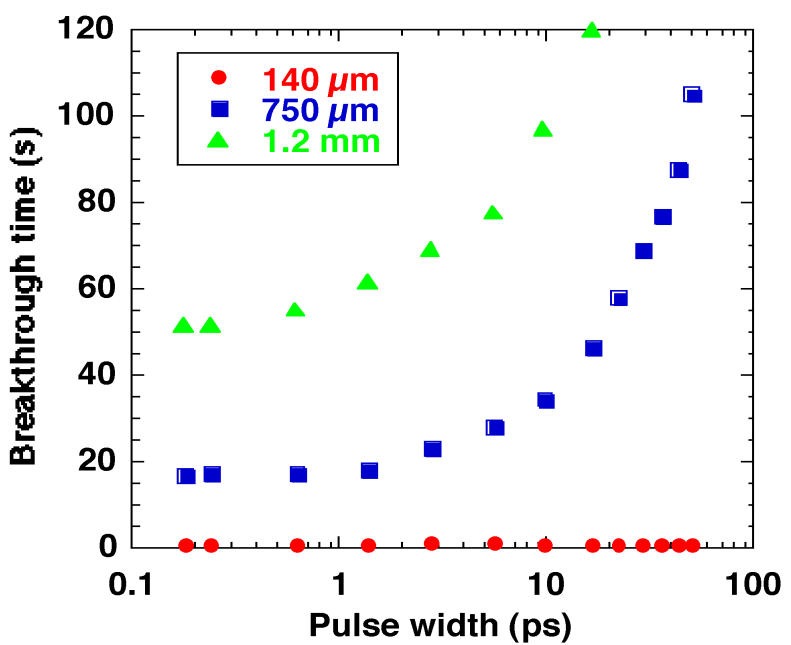

Fig 5b : Experimentally observed breakthrough times for stainless steel, $45 ;$ AOI, $825 \mathrm{~nm}$, circular polarization, 8 $\mathrm{J} / \mathrm{cm}^{2}$.

The results of the simulations are consistent with the experimental data presented in Fig.5b. The time needed to drill through films of varying thicknesses of stainless steel was measured. One can see that for pulses shorter than 1 psec the time to drill through is insensitive to pulse duration and that drilling time goes up sharply for pulses longer than 10 psec.

Experimentally, the ablation rate s functional dependence on absorbed energy becomes more complicated. As the machined holes or slots develop, the bottom surface becomes corrugated as illustrated in Fig. 6. Possible causes of this will be discussed later in section 4. Nonetheless, this surface structure will most likely affect the fraction of energy absorbed at the ablation surface. Evidence of this is seen in the fact that the depth of holes drilled in aluminum and copper are four and five times, respectively, that of holes drilled in stainless steel for the same number of laser pulses. This is in spite of the fact that the expected absorbed energy in copper, calculated from the Fresnel coefficients, is $1 / 10$ that of stainless steel. ${ }^{11}$ The various features also exhibit different dependence on incident fluence, making it difficult to correlate the observed removal with a 1-d planar surface approximation.

\section{ABLATION PLUME EXPANSION}

For laser film deposition and laser breakdown diagnostics, it is desirable to relate parameters of ejecta far from the target to those near the target. The large variety of physical processes involved, for instance laser-material interaction, hydrodynamics, thermal and radiative transport, make an analytical description extremely difficult. Numerical simulations seem to be the only viable option. However, the large disparity in spatial and temporal scales involved make it impractical to simulate the ejecta expansion to large distances from the target within the framework of the radiation-hydrodynamic ablation model. For example, a typical calculation would require an adequate description of the laser energy deposition that occurs on a time scale of hundreds of femtoseconds and spatial distances on the order of tens of nanometers, while the same model would be required to predict the behavior of ejecta at the final stage of expansion that takes place microseconds later and centimeters away from the initial energy deposition event.

A possible solution to this problem is to employ more than one model, each one suitable for a particular stage, and to match them at an intermediate time. Plume evolution includes two important parts - an initial one-dimensional and a final threedimensional evolution. The difference is not only geometrical, but in the type of physics playing a major, defining role. The one-dimensional stage takes place during the time of actual laser energy deposition when the initial expansion occurs. At this time the longitudinal size of the hot material is still much smaller than the transverse size which is set by the laser spot size. Laser-material interaction, ionization, energy and material transport are the dominating physics during this period. After some time the ejecta cloud becomes truly three-dimensional, and the physics simplifies considerably. At this stage, conventional hydrodynamics plays the dominant role and plume evolution is sensitive only to integral parameters of the laser pulse such as total energy and mass. Anisimov et.al. ${ }^{12}$ suggested an exact hydrodynamic similarity solution for $3 \mathrm{D}$ gas ellipsoid expansion to describe such ejecta. In this model, the plasma is treated as an adiabatically expanding ellipsoidal 


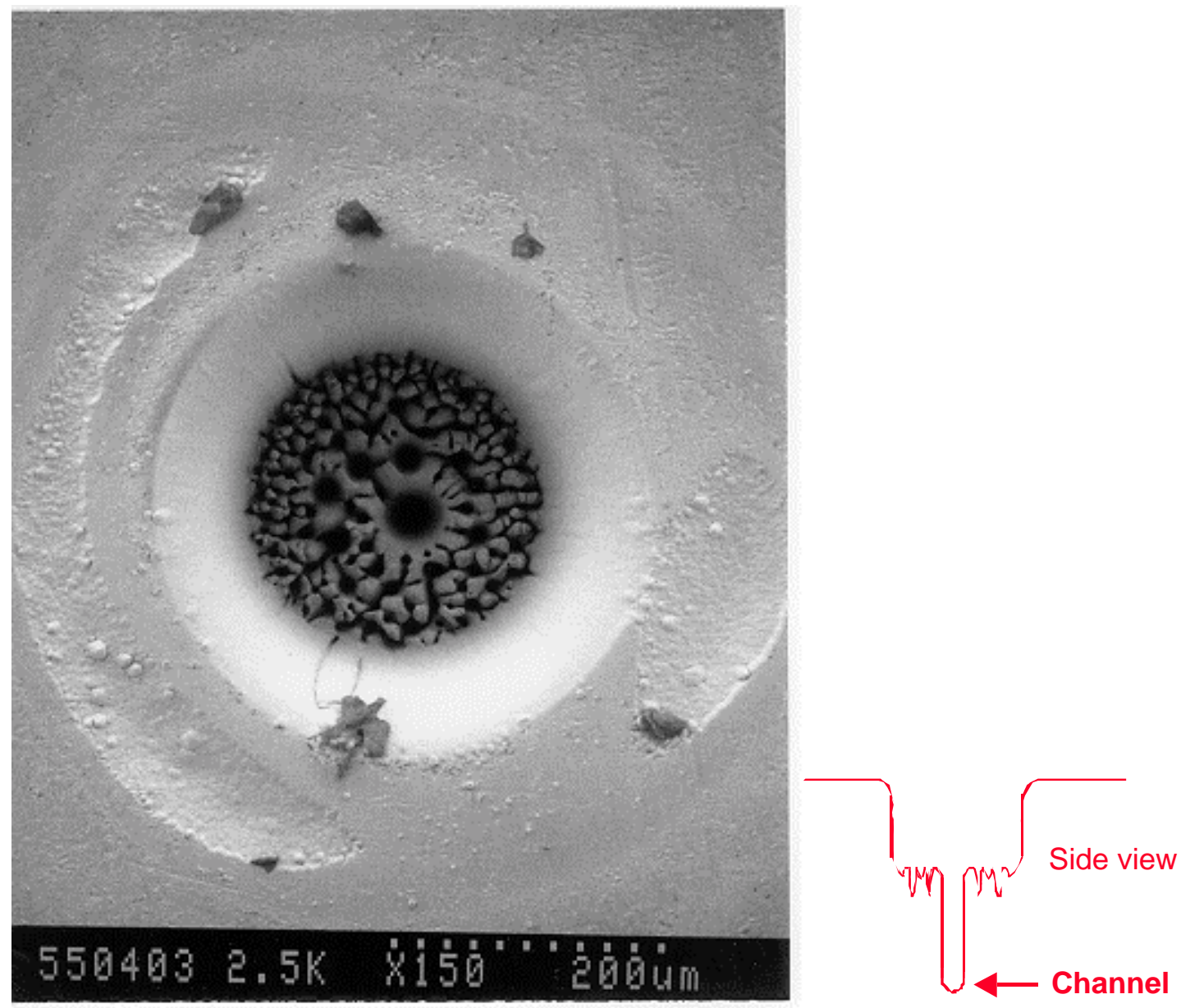

Fig. 6. SEM micrograph of hole drilled for 6000 shots with a gaussian spatial profile at normal incidence in stainless steel, 1 ps pulse length, circular polarization, $10 \mathrm{~J} / \mathrm{cm} 2$. The inset is a rough depiction of how the cross-section would look.

cloud. All flow parameters are constant on ellipsoidal surfaces for this solution. The expansion process description includes gas cooling and cloud shape deformation.

The expansion of the plasma ellipsoid is determined by six parameters-the initial ellipsoid sizes, plume mass and energy and gas adiabatic constant. The spot size can be taken as the ellipsoid initial transversal size. The zero velocity position introduced above is considered as the boundary of expanded material; the mass and energy of ejecta constitute half of the ellipsoid s mass and energy (other half of ellipsoid is inside the material).

The thickness of removed material can be taken as the initial longitudinal size. Calculations indicate that plume properties are not very sensitive to the exact value of initial thickness. Finally the ideal gas adiabatic constant of 5/3 was assumed. As a result we are able to characterize the plume parameters (energy, density and velocity distribution) in terms of laser radiation and target parameters. Fig. 7 shows the calculated energy of the plume as a function of absorbed energy. One can see that plume energy and absorbed laser energy are proportional for a wide range in absorbed energy; about $90 \%$ of the absorbed energy is ejected with the plume. This is consistent with the small collateral damage observed in USLP processing.

Initially, the heated material has a pancake shape with transverse size much larger than longitudinal size. Pressure changes slowly along the surface, but sharply across the ablated surface. As a result, material is mainly accelerated normal to the surface and plume expansion is very directed. The calculation of plume angular width versus pulse intensity for different spot sizes is shown in Fig. 8. The bumps on the curve probably are of numerical nature. 


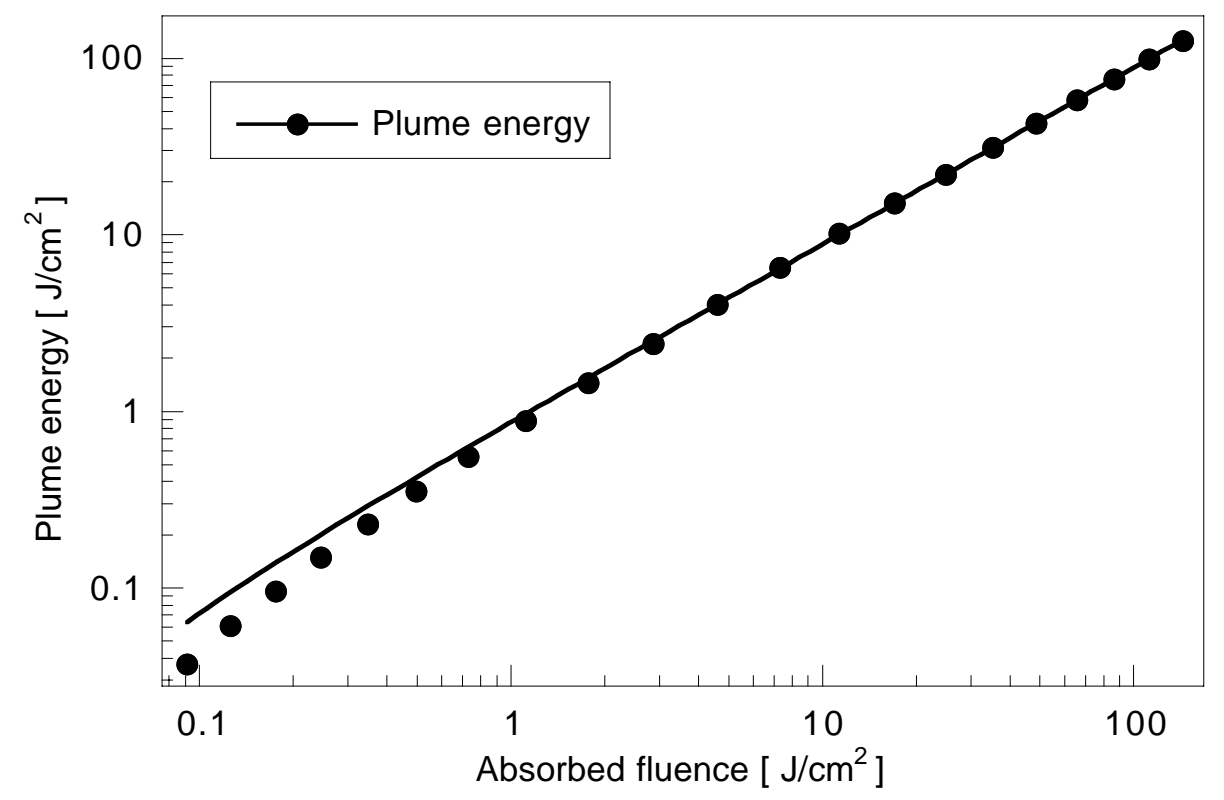

Fig 7 : Calculated plume energy as a function of absorbed fluence ( Aluminum, normal incidence, $\lambda=826 \mathrm{~nm}, \tau=150 \mathrm{fs}$ ).

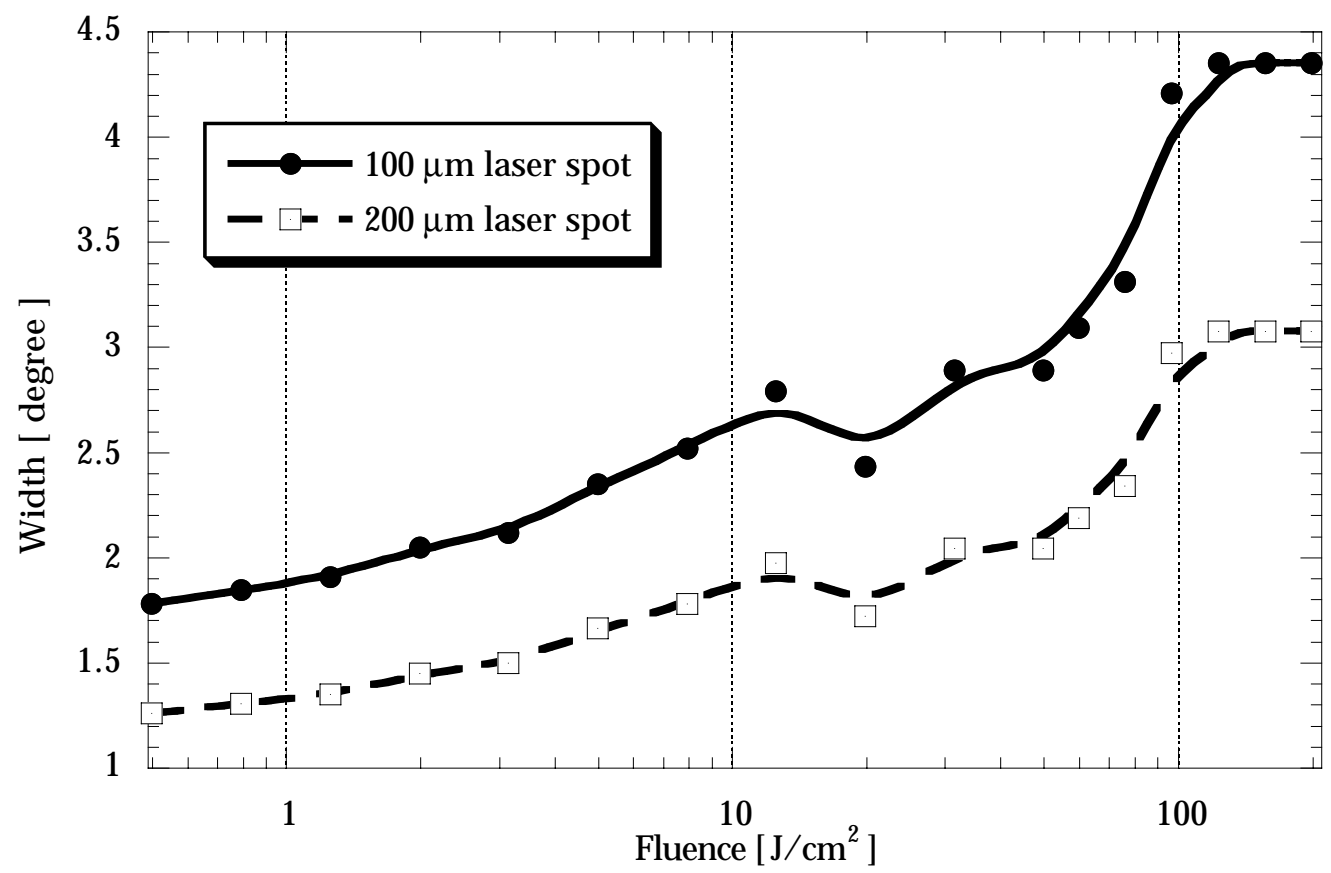

Fig.8. Calculated plume width (FWHM) as a function of fluence for two laser spots. Material is aluminum. Laser parameters : $\lambda=826 \mathrm{~nm}, \tau=150 \mathrm{fs}, \theta=45^{\circ}$, $\mathrm{S}$ polarization. 


\section{GROWTH OF SURFACE RELIEF}

The crater bottom can become corrugated soon after material removal begins. Surface relief developed over many shots is shown in Fig.9. Since each individual shot removes only a small amount of material, drilling a hole through a film thicker than the hole diameter encounters the ablation of corrugated surfaces.

A plane metal surface irradiated by laser light can be unstable to the growth of surface relief. Such instability can be understood as follows (see e.g. Refs. 13, 14). Consider a small periodic surface modulation (grating) with wave number $\mathbf{g}$. Surface modulations diffract the incident wave, and the interference of the incident and diffracted waves produces a periodic variation of light energy absorption. If this modulation of absorbed energy leads to growth of the grating, it serves as a feedback mechanism driving the growth of surface modulations.

The problem of surface relief excitation has been considered in the literature mainly in the context of CW or long laser pulses. Several feedback mechanisms have been investigated. ${ }^{14}$ First, evaporation should be mentioned. Higher local intensity results in increased surface temperature which, in turn, enhances the evaporation producing relief growth. Other mechanisms studied included the thermocapillary mechanism and the melt displacement by vapor reciprocal momentum. ${ }^{14}$ All these mechanisms are irrelevant to the USLP interaction with metals.

In our case, the deposited energy density is very high. Local pressures can reach the megabar range. Energy transfer occurs not only via thermal conduction, but also by shockwaves. Nevertheless, the immediately affected layer of material is very thin and remaining material solidifies very fast after pulse termination, on a time scale of $0.1-1 \mathrm{nsec}$. The melt solidifies before any relief relaxation can take place. ${ }^{15}$ Instability development takes place during the pulse for long pulses. For USLP, the cooling time is too fast. Surface relief modulations survive from one shot to the next; optimal small surface variations accumulate with the following pulses, and relief formation takes place after many shots.

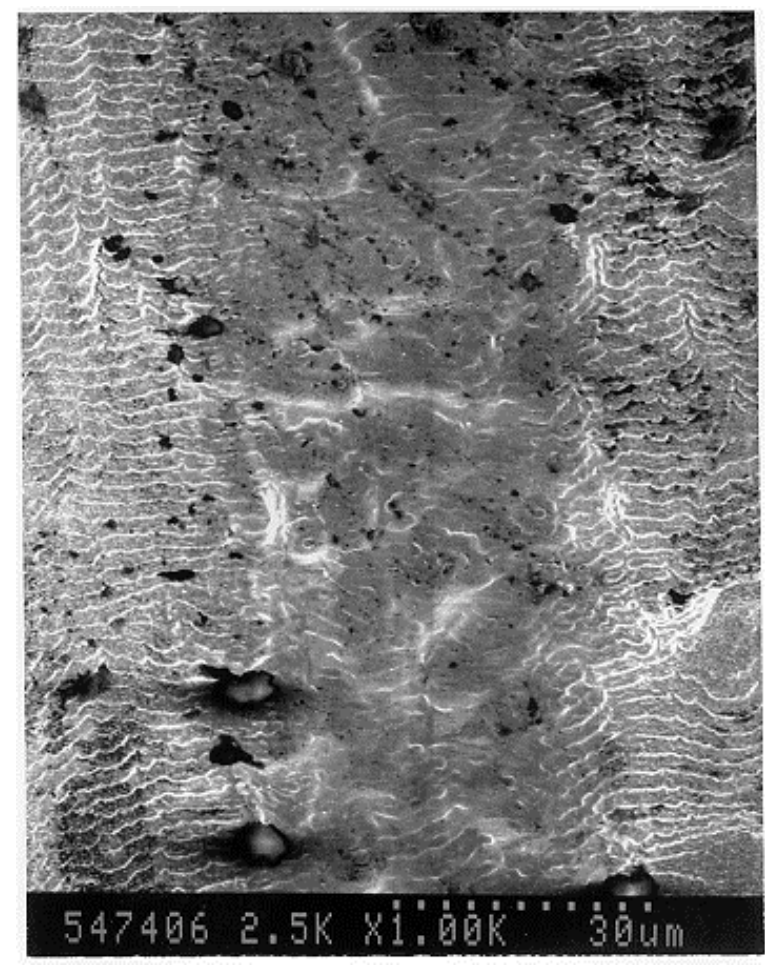

(a)

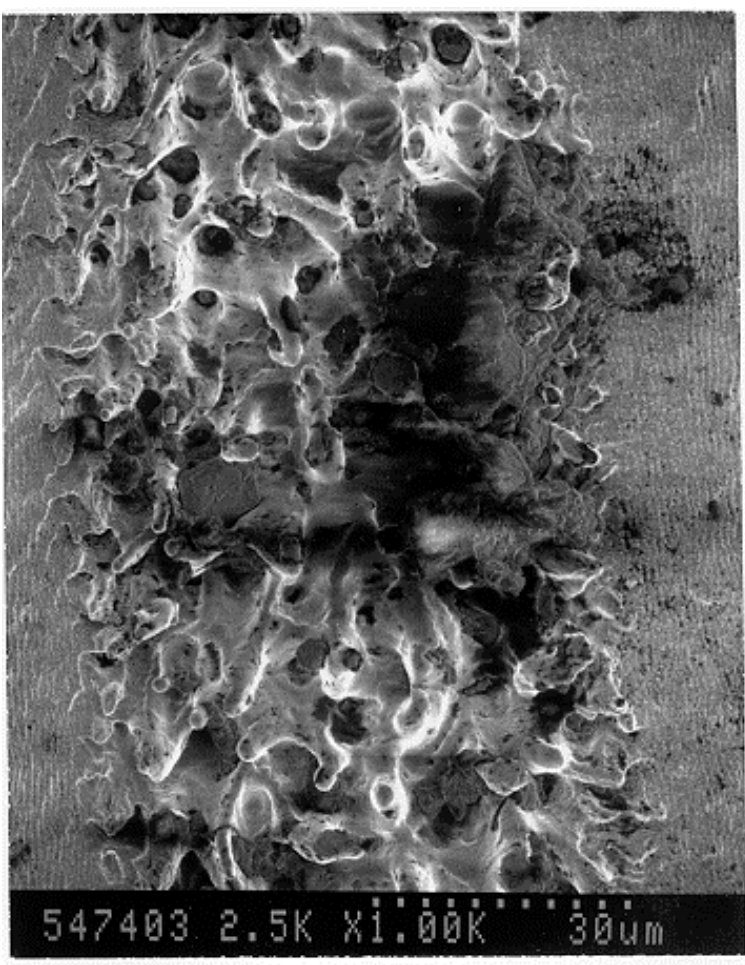

(b)

Fig. 9. SEM photomicrographs of surface of holes after 200 pulses drilled in stainless steel at $45 i$ angle of incidence and $8 \mathrm{~J} / \mathrm{cm}^{2}$ for P (a) and S (b) linear polarization. The plane of incidence is vertical in these pictures. 
For USLP shorter than a few psec, material removal is expected to be determined by the locally absorbed energy Q and is not sensitive to the pulse duration. Absorbed energy $Q$ is, in turn, determined by the pulse energy, the material absorption coefficient, and the polarization and angle of incidence of the light. The thickness of material removed by one pulse can be written as

$$
\frac{d z}{d N}=F(Q)
$$

Here $\mathrm{z}=\mathrm{Z}_{0}+\mathrm{Z}_{1}$ is the surface position, $\mathrm{Z}_{0}$ is the spatially averaged value, while $\mathrm{z}_{1}$ is the local corrugation. $\mathrm{N}$ is the number of laser shots and $\mathrm{F}$ is a monotonically increasing function of energy $\mathrm{Q}$ to be defined either theoretically or empirically. Interference of the incident and scatterred waves results in modulation of absorbed energy: $Q=Q_{0}+Q_{1_{-}}$which produces the feedback necessary for relief growth..

It is important to mention that the wavelength of the most unstable relief is non-sensitive to the feedback mechanism. It is evident that the light interaction with the surface modulation will be most efficient when the scattered wave propagates along the surface. For this electromagnetic surface wave we must have

$$
\begin{aligned}
& \mathbf{k}_{t} \pm \mathbf{g}=\mathbf{k}_{s} \\
& \left|\mathbf{k}_{t}\right|=k \sin (\vartheta) \\
& \left|\mathbf{k}_{s}\right|=k
\end{aligned}
$$

The maximal growth has structures in which the tangential part of the incident laser electric field will be parallel to the Bragg vector g. For P-polarization, the relief wavelength is given by [see e.g. Ref. 14]

$$
\lambda_{s}=\frac{\lambda}{1 \pm \sin \vartheta}
$$

For an angle of incidence of 45 degrees, the relief wavelength is about 3.4 times the laser wavelength. For S-polarization, the surface modulation wavelength is given by

$$
\lambda_{s}=\frac{\lambda}{\cos \vartheta}
$$

and is 2.4 times shorter. From fig. 9, one can see that both the orientation and wavelength of surface relief observed in experiments are consistent with the above estimates.

We estimate now the number of pulses needed for relief development. Separating the CW and oscillating parts of (1) we have

$$
\begin{aligned}
& \frac{d Z_{0}}{d N}=F\left(Q_{0}\right) \\
& \frac{d z_{1}}{d N}=\frac{d F}{d Q} Q_{1}
\end{aligned}
$$

The periodically varying energy absorption is evaluated in, e.g., Refs. 14,15. For wave vectors satisfying (4.2) we have

$$
\begin{aligned}
& Q_{1}=Q_{0} \operatorname{Im} \frac{1}{\xi} A k z_{1} \\
& A=\frac{\left|E_{p} \cos \varphi+E_{s} \sin \varphi \cos \vartheta\right|^{2}}{\left|E_{p}\right|^{2}+\left|E_{s} \cos \vartheta\right|^{2}}
\end{aligned}
$$


Here $\mathrm{E}_{\mathrm{p}}$ and $\mathrm{E}_{\mathrm{s}}$ are $\mathrm{P}$ and $\mathrm{S}$ polarization components of the laser field, $\boldsymbol{\varphi}$ the angle between the vectors $\mathbf{k}_{\mathbf{t}}$ and $\mathbf{k}_{\mathbf{t}}+\mathbf{g}$, and $\boldsymbol{\xi}$ the surface impedance: $\xi=\frac{1}{\sqrt{\varepsilon}}<<1$. For resonantly excited relief with maximal growth rate $\mathrm{A}=1$, one can see that (5a) and $(5 b)$ describes exponential growth of the relief amplitude with the number of pulses.

$$
\begin{aligned}
z_{1} & =z_{1}(0) \exp G \\
G & \approx \int d N \frac{d F}{d Q} Q_{0} \operatorname{Im} \frac{k}{\xi}
\end{aligned}
$$

Our simulations above indicated that for $\mathrm{Al}$ ablation, $\mathrm{F}$ is a power function of $\mathrm{Q}$ Approximating the function $\mathrm{F}$ as a power $\mathrm{m}$ of the absorbed energy Q, G can be estimated as

$$
G \approx m \operatorname{Im}(1 / \xi) k Z_{0}>k Z_{0}
$$

where $\mathrm{Z}_{0}$ is the average depth of material removed after $\mathrm{N}$ pulses

This type of periodic relief shown in Fig. 9 is only observed for low incident laser fluence or in the low fluence region of a more energetic Gaussian pulse. At higher fluences (above $.5 \mathrm{~J} / \mathrm{cm}^{2}$ in stainless steel), deeper modulation of the surface of the hole bottom is observed with scale lengths of tens of wavelengths. This can be seen in the center region of the hole shown in Fig. 9(b) as well as in the more developed holes shown in Figs. 6 and 10. The depth of this modulation grows with increasing fluence as well as with increasing number of pulses. Some of the features seen in Fig. 9(b) actually extend above the original surface, indicating that redeposition of material also plays a role in the formation of these structures

The smoothing is probably related to the dependence of the local heat of evaporation on local surface curvature

$$
L_{e f f}=L-(\sigma / \rho)(1 / R)
$$

where $\mathrm{L}$ is the specific heat of evaporation for a planar boundary, $\sigma$ is the surface energy density (surface tension), and $\mathrm{R}$ is the radius of curvature of the boundary. At high energy pulse ablation the small scale structures are eliminated by evaporation and longer scales structures are generated. The development of long-wave surface modulation is enhanced by changes of absorption due to local variation of polarization and angle of incidence.

As mentioned above, these features are formed over several hundred or thousand pulses with only a few tens or hundreds of nanometers of material being removed per pulse. We have seen no correlation between the surface modulation of the hole bottom to spatial variations in the laser beam, but successive holes drilled under the same conditions exhibit very similar morphology. There must be a stable mechanism which seeds these surface instabilities as well as a feedback mechanism over many pulses which leads to their growth and the resulting deep modulation. This mechanism does appear to depend on material properties as can be seen in the differences in the morphology between stainless steel, aluminum, and copper shown in Figs. 6 and 10.

Of particular interest in the features seen in Figs. 6 and 10(a) is the circular hole that seen near the center of these two larger holes. Above a certain threshold fluence (approximately $5 \mathrm{~J} / \mathrm{cm}^{2}$ in stainless steel and aluminum), such circular channels are always observed for holes deeper than 50-100 m, with the depth after a given number of pulses increasing rapidly with increasing fluence. It appears that above this threshold fluence, one of the smaller features near the peak of the fluence begins to enlarge and grows in depth faster than the surrounding features. This deep channel tends to have nearly perfect circular symmetry with smooth walls and diameters at the top of the channel ranging from 30 to $100 \mathrm{~m}$. The channels can also extend several millimeters into the material with the removal rate, once the channel is formed, being approximately independent of depth. Finally, we have observed the formation of these channels even when doing line cuts where the beam is scanned across the surface. In this case, a series of these channels approximately $30 \mathrm{~m}$ in diameter was formed in a quasiperiodic row at the bottom of the slot. The period of formation $(\sim 50 \mathrm{~m})$ is independent of spot size or scan rate (which was slow enough to have significant beam overlap from pulse to pulse).

We have not yet determined what parameters affect the creation and growth of these deep channels during hole drilling, but there is no apparent correlation with the incident laser spot except that the channel will always appear near the peak of the fluence, i.e. for a Gaussian beam, the channel will be near the center while for an annulus, there will be a ring of channels in the outer region of the hole. The channel diameter increases only slightly with larger fluences but is larger in aluminum then 


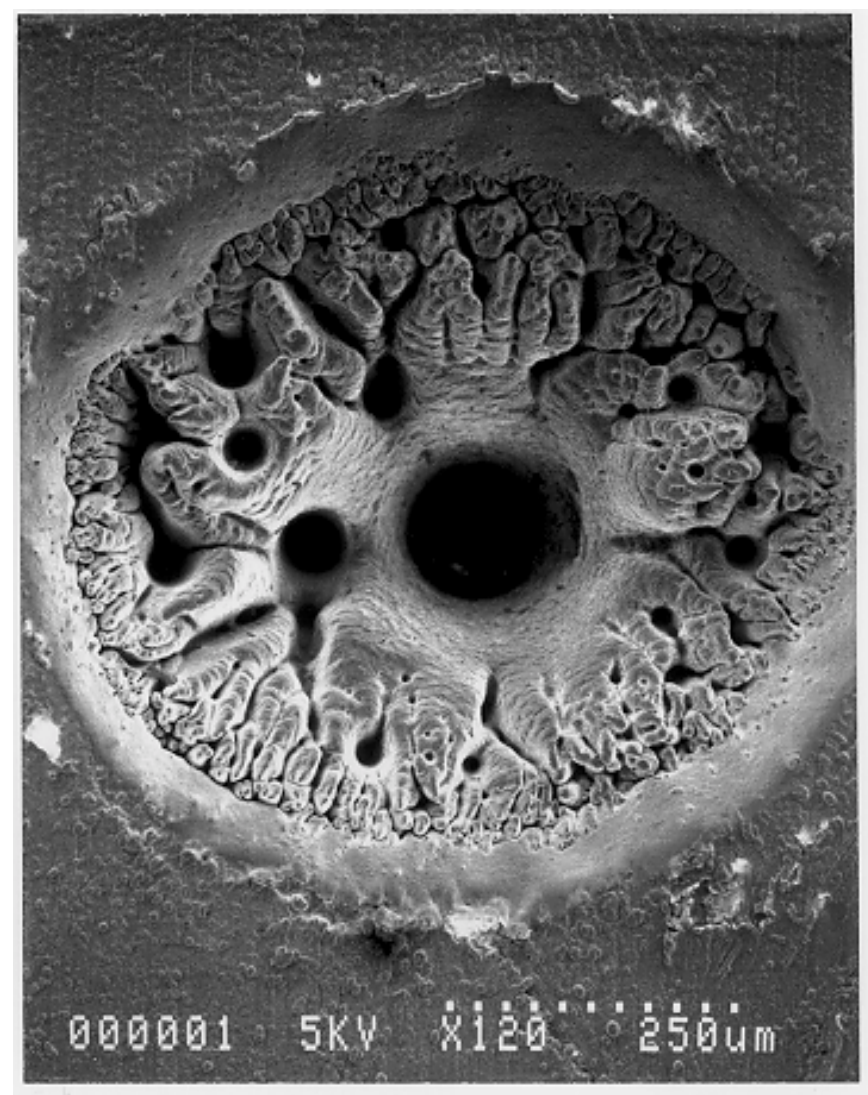

(a)

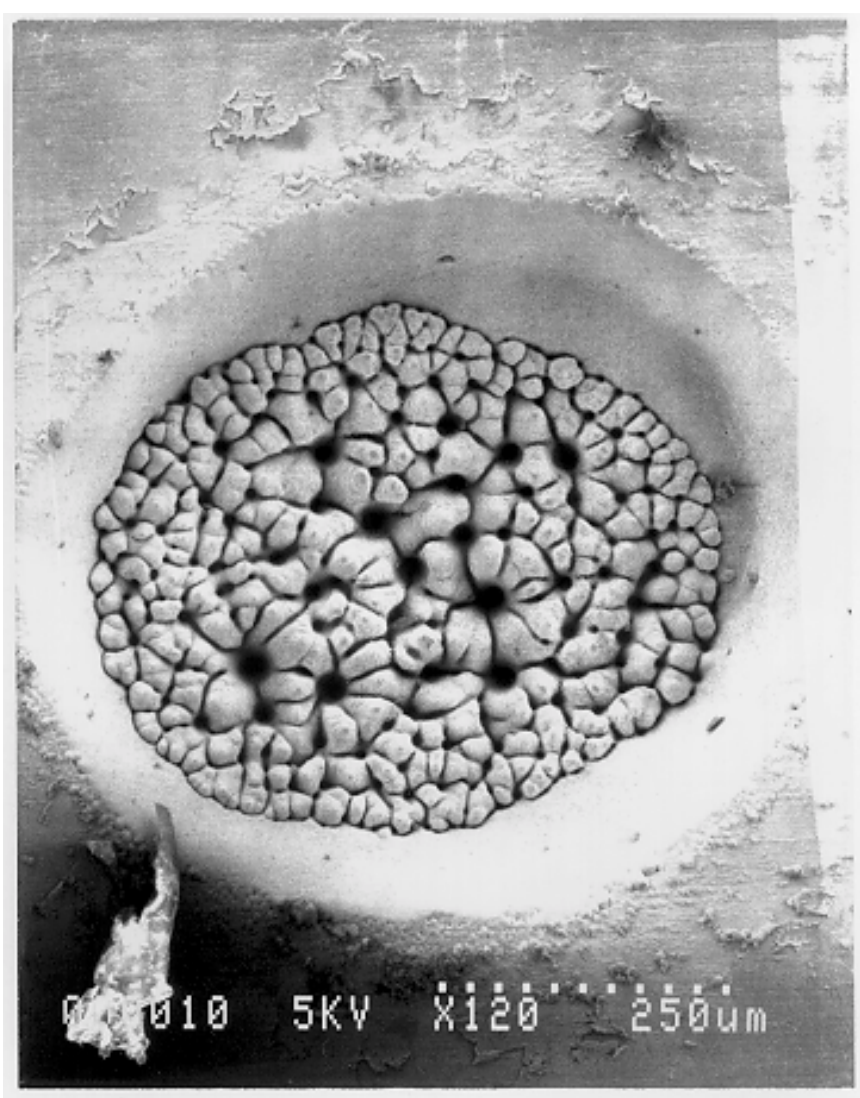

(b)

Fig. 10. SEM photomicrographs of blind holes drilled in (a) aluminum and (b) copper at $15 \mathrm{~J} / \mathrm{cm}^{2}$ after 1500 shots. The laser parameters were normal incidence, circular polarization, and $1 \mathrm{ps}$ pulse length.

in stainless steel. There is also some dependence of the channel diameter on the overall size of the hole being drilled, which we have not yet quantified. In addition, while ablation of much of the surrounding material is independent of fluence, the material removed per pulse inside this central channel increases linearly with fluence and can reach rates that are several times the rate of material removal in the larger hole. In practical applications, this means that at high fluences, energy will break through the work piece through this channel much sooner than the completion of the removal of the surrounding material. This makes reporting of drilling rates dependent on how the rate was determined.

However, material properties also have an effect on the formation and growth of the deep channel features. In fact, when drilling holes in copper (Fig. 10(b)), we have observed no large, deep channels for fluences up to $15 \mathrm{~J} / \mathrm{cm}^{2}$. Instead, many smaller channels ( $\sim 10-20 \mathrm{~m}$ diameter) are formed, but these do not extend as deeply into the material. The ablation rate inside these channels also increases more slowly with fluence than those discussed above for aluminum or stainless steel. We have not yet determined if large, deep channels can occur in copper at higher fluences, nor have we determined what property of copper contributes to the suppression of the deep channels.

The presence of deep surface modulation in turn modifies the laser material interaction. We found above that, for ablation of a plane, about $90 \%$ of the absorbed energy is carried out by the plume, which is ejected normal to the surface. The melted layer is very thin and solidifies before any surface modification takes place. When laser radiation impinges on a corrugated surface, however, ejected material can hit the side of a nearby crest. Redeposition of energy, mass and momentum then takes place. The typical flight time for relief with height scale of tens of microns is in the nanosecond range. Hence, more absorbed energy now returns to the condensed material, and the energy deposition time now stretches up to a few nanoseconds. The resultant melt is deeper and has longer time for evolution. In addition, the deposited momentum drives the melt motion and can produce the structures observed in experiments. 


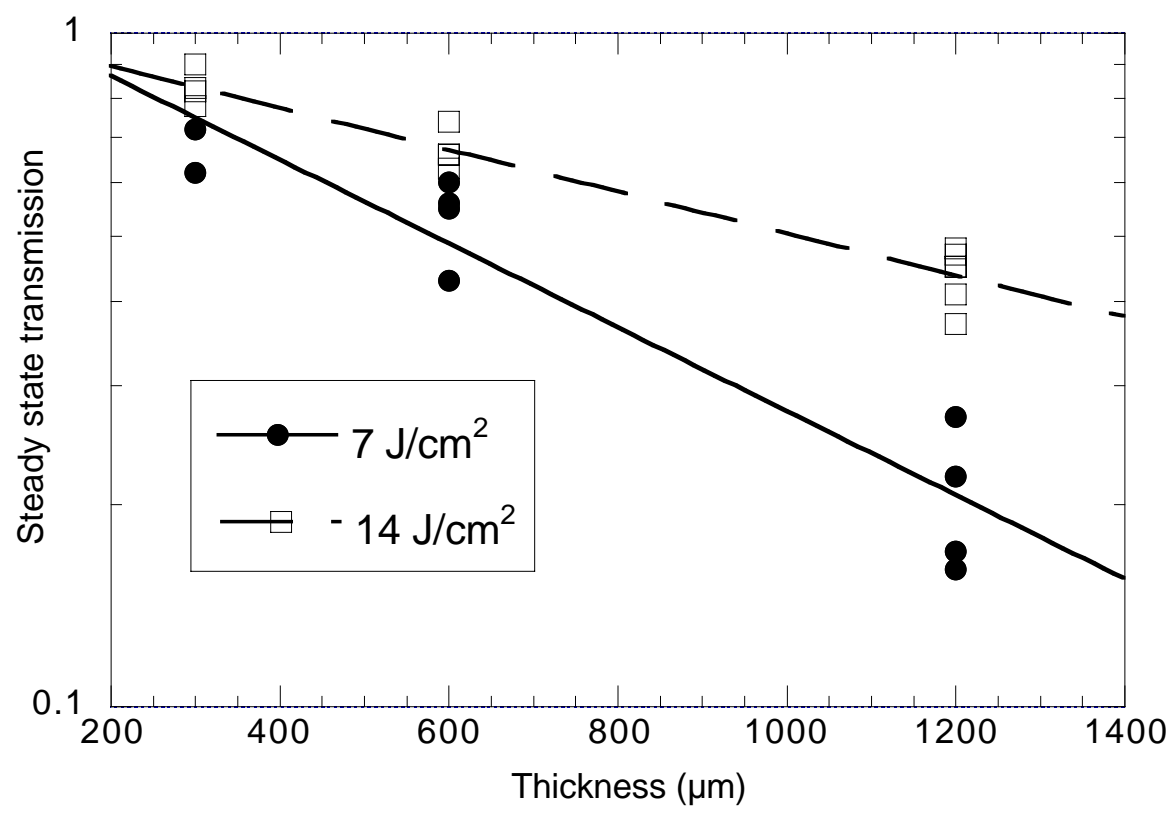

Fig. 11 : Measurements of a weak signal transmission through holes drilled with a higher fluence and the same spatial profile in stainless steel plates of various thickness.

\section{THE INTERACTION OF RADIATION WITH THE WALLS.}

Applications often require drilling a deep hole with high aspect ratio. Laser light propagating toward the bottom of a deep hole can be appreciably attenuated and can undergo changes in spatial distribution. This has a strong affect on hole morphology. Fig. 11 presents weak signal measurements of transmission through a hole drilled at higher intensity with the same beam shape. A significant fraction of the laser energy is absorbed or scattered before reaching the bottom. Holes drilled through a fixed thickness at higher intensity are wider and transmit more light.

To evaluate absorption in the walls consider the propagation of light in a straight cylindrical channel with radius a. For numerical estimates we consider drilling of $\mathrm{Al}$ with 0.8 micron radiation. The dielectric constant for $\mathrm{Al}$ at this wavelength is $\varepsilon=-61.5+45.7 \mathrm{i},{ }^{16}$ and the typical hole radius $\mathrm{a}=100 \mathrm{~m}$. The similar linear problem of $\mathrm{CO}_{2}$ laser propagation in metallic capillaries was studied in [17] and we will adopt their results for our case.

For ^ $\varepsilon<<$ ka modes with TE and TM polarization are strongly coupled. Consider only angular modes with $\mathrm{m}=1$ and longitudinal propagation number $\mathrm{h}$ corresponding to the linear polarization of incident radiation, $\mathrm{E}, \mathrm{H} \sim \mathrm{f}(\mathrm{r}) \exp (\mathrm{i} \phi+\mathrm{ihz})$. For typical drilling experiments, the condition $k_{\perp}^{4} a^{2}|\varepsilon|<<4 k^{2}$ is valid, where the transverse wavenumber $k_{\perp}$ is $k^{2}-h^{2}$ and TE and TM modes are mixed. For a hybrid mode propagating along the channel the damping coefficient is given by expression.

$$
\alpha_{E H} \sim \frac{k_{\perp}^{2} \varepsilon^{\prime \prime}}{8 \pi k^{2} a \sqrt{|\varepsilon|}}
$$

For the lowest mode with minimal damping

$$
\begin{aligned}
& k_{\perp}=\frac{\pi}{a} \\
& \alpha_{E H} \sim \frac{\pi \varepsilon^{\prime \prime}}{8 k^{2} a^{3} \sqrt{|\varepsilon|}}
\end{aligned}
$$


The corresponding expression for higher order modes is multiplied by $\mathrm{m}^{2}$ where $\mathrm{m}$ is the mode number. For the typical drilling conditions indicated above, the damping of the fundamental and lower modes is thus expected to be very small, the attenuation length being over $10^{5}$ times the hole radius. Experiments ${ }^{17}$ carried out for $\mathrm{CO}_{2}$ radiation propagation in capillaries, however, discovered much higher damping. Even polished surfaces of capillaries were corrugated enough to transfer significant energy from the volume mode to heavily damped surface waves with

$$
\alpha_{s} \approx \frac{k \varepsilon^{\prime}}{2|\varepsilon|^{2}}
$$

on a scale less than the hole radius. Surface roughness in the wall of drilled holes is surely larger than that of polished waveguides so strong resultant attenuation is natural. The instability of surfaces to corrugation growth discussed above is expected to take place for the hole walls, but we presently have no experimental observation of this. Additional corrugations can be produced by a material re-deposition. The nonlinear stage of corrugation growth at high fluence is presently not understood.

One of the most intriguing phenomena associated with deep drilling is the formation of smooth round channels as descrbed in Sec. 4. Radiation absorption in the walls possibly plays a role in the formation of such channels. For small channels, absorption is strong enough to remove wall corrugations by evaporation. This "ablative polishing" is expected to ultimately result in a decrease in channel absorption. For a wide channel, absorption in the walls is not sufficient to heat material to melting. For the experimental conditions corresponding to Fig. 10, for example, estimates indicate that even if all nontransmitted energy were absorbed in the walls homogeneously over the full length of the hole, this energy is insufficient to cause melting of the surface. For a very small channel, absorption is strong even for smooth walls and radiation cannot propagate far. We can speculate that there may be an optimal channel size at which ablative polishing yields smooth walls without being so small as to result in strong damping.

\section{CONCLUSION}

There is a rich set of processes involved in the ultrashort-pulse laser drilling of metals. We understand reasonably well the ablation of planar metal surfaces. Unfortunately for processing applications, ablation of planar surfaces is unstable to the growth of surface corrugation. We demonstrated that corrugations become significant after removal of a layer of thickness only a few laser wavelengths. Corrugations change the laser light energy deposited in the material. Light absorption in the walls plays an important role in drilling of deep holes. Also re-deposition of the material can affect the absorption in the walls and structure formation on the crater bottom. Self-organized coherent structures form on the bottom of the crater. Even good qualitative understanding of these latter phenomena is presently lacking.

\section{ACKNOWLEDGEMENTS}

This work was performed under the auspices of the US Department of Energy by the University of California Lawrence Livermore National Laboratory under contract No. W-7405-ENG-48.

\section{REFERENCES}

1. S. Preuss, A. Demchuk, and M. Stuke: Appl. Phys. A 61, 33 (1995).

2. B.N. Chichkov, C. Momma, S. Nolte, F. von Alvensleben, and A. T nnermann: Appl. Phys. A 63, 109 (1996).

3. P. S. Banks, B. C. Stuart, M. D. Perry, M. D. Feit, A. M. Rubenchik, J. P. Armstrong, H. Nguyen, F. Roeske, R. S. Lee, B. R. Myers, and J. A. Sefcik: in Conference on Lasers and Electro-Optics, Vol. 6 of Technical Digest Series (Optical Society of America, Washington, D. C., 1998), paper CFD2.

4. M. D. Perry, B. C. Stuart, P. S. Banks, M. D. Feit, V. Yanovsky, and A. M. Rubenchik: J. Appl. Phys. 85, 6803 (1999).

5. D. Ashkenasi, A. Rosenfeld, H. Varel, M. W hmer, and E. E. B. Campbell: Appl. Surf. Sci. 120, 65 (1997).

6. M. Lenzner, J. Kr ger, W. Kautek, and F. Krausz: Appl. Phys. A 68, 369 (1999).

7. T. Her, R. J. Finlay, C. Wu, S. Deliwala, and E. Mazur: Appl. Phys. Lett. 73, 1673 (1998).

8. J. T. Larsen, "HYADES - A Radiation Hydrodynamics Code for Dense Plasma Studies," in Radiative Properties of Hot Dense Matter, edited by W.Goldstein, C. Hooper, J. Gauthier, J. Seely and R. Lee, World Scientific, Singapore, 1991, A.M.Rubenchik, M.D.Feit, M.D.Perry, J.T.Larsen Appl. Surf. Sci. 127-129, 193 (1998). 
9. A.M. Komashko, M.D. Feit, A.M. Rubenchik, M.D. Perry, P.S. Banks, Simulation of material removal efficiency with ultrashort laser pulses, Appl. Phys. A 69, pp. S95-S98, 1999.

10. H. M. Milchbegr, R. R. Freeman, S. C. Davey, R. M. More, "Resistivity of a simple metal from room temperature to $10^{6} \mathrm{~K} "$, Phys. Rev. Lett. 61, pp. 2364-2367, 1988.

11. P. S. Banks, B. C. Stuart, A. M. Komashko, M. D. Feit, A. M. Rubenchik, and M. D. Perry, Femtosecond Laser Materials Processing, Proc. SPIE 3934, 2000.

12. S.I. Anisimov, B.S. Luk'yanchuk and A. Luches, "An analytical model for three-dimensional laser plume expansion into vacuum in hydrodynamic regime", Applied Surface Science 96-98, pp. 24-32, 1996.

13. S.I.Anisimov, V.A.Khokhlov, "Instabilities in Laser-Matter Interaction" CRC Press, 1995.

14. S.A.Akhmanov, V.I.Emelyanov, N.I.Koroteev, V.N.Seminogov, "Interaction of powerful laser radiation with the surfaces of semiconductors and metals: nonlinear optical effects and nonlinear optical diagnostics", Sov.Phys.-Uspekhi, 28, 1084, 1985.

15. M.B. Agranat, S.I. Ashitkov, V.E. Fortov, S.I. Anisimov, A.M. Dykhne, P.S. Kondratenko, "Formation of periodic surface structures by ultrashort laser pulses", JETP 88, pp. 370-376, 1999.

16. Handbook of Optical Constants of Solids, Ed. by Palik,E.D.,Academic press, New York, 1991.

17. V.N.Anisimov L.A.Bolshov, K.A.Krivoruchko, D.D.Malyuta, V.P.Reshetin, A.Yu.Sebrant, R.I.Soloukhin, "Absorption of infrared radiation in metallic capillaries," Sov.J.Quantum Electronics 17, 101, 1987. 